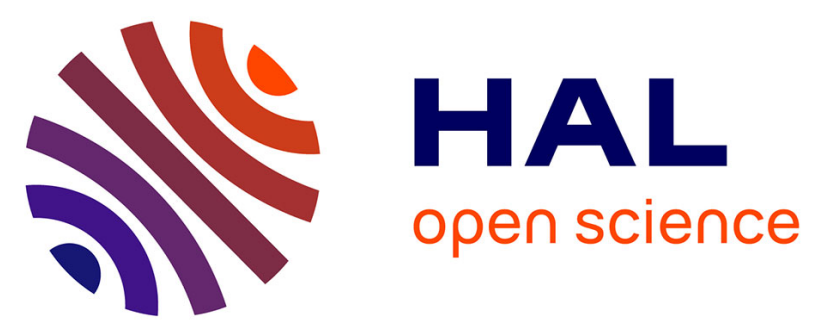

\title{
Les élections européennes du 13 juin 1999 en Languedoc-Roussillon : de la nationalisation du vote à la régionalisation des particularismes
}

Paul Alliès, François Baraize, Emmanuel Négrier, Philippe Secondy

\section{- To cite this version:}

Paul Alliès, François Baraize, Emmanuel Négrier, Philippe Secondy. Les élections européennes du 13 juin 1999 en Languedoc-Roussillon: de la nationalisation du vote à la régionalisation des particularismes. Pôle Sud - Revue de science politique de l'Europe méridionale, 1999, Enjeux migratoires en Europe du Sud, 11, pp.97-115. 10.3406/pole.1999.1055 . hal-02484111

\section{HAL Id: hal-02484111 \\ https://hal.umontpellier.fr/hal-02484111}

Submitted on 28 Apr 2020

HAL is a multi-disciplinary open access archive for the deposit and dissemination of scientific research documents, whether they are published or not. The documents may come from teaching and research institutions in France or abroad, or from public or private research centers.
L'archive ouverte pluridisciplinaire HAL, est destinée au dépôt et à la diffusion de documents scientifiques de niveau recherche, publiés ou non, émanant des établissements d'enseignement et de recherche français ou étrangers, des laboratoires publics ou privés.

\section{(2)(1) $\$$}

Distributed under a Creative Commons Attribution - NonCommercial - NoDerivatives $\mid 4.0$ 


\section{Les élections européennes du 13 juin 1999 en Languedoc-} Roussillon : de la nationalisation du vote à la régionalisation des particularismes

Mr Paul Alliès, Mr François Baraize, Mr Emmanuel Négrier, Mr Philippe Secondy

\section{Citer ce document / Cite this document :}

Alliès Paul, Baraize François, Négrier Emmanuel, Secondy Philippe. Les élections européennes du 13 juin 1999 en Languedoc-Roussillon : de la nationalisation du vote à la régionalisation des particularismes. In: Pôle Sud, n¹1, 1999. Enjeux migratoires en Europe du Sud. pp. 97-115;

doi : https://doi.org/10.3406/pole.1999.1055

https://www.persee.fr/doc/pole_1262-1676_1999_num_11_1_1055

Fichier pdf généré le 23/04/2018 


\section{Les élections européennes du 13 juin 1999 en Languedoc-Roussillon :}

de la nationalisation du vote à la régionalisation des particularismes

\section{par Paul Alliès, François Baraize, Emmanuel Négrier, Philippe Secondy Cartographie : François Baraize Póle Sud $N^{\circ} 11$-novembre 1999 -p. 97 à 115}

Les élections européennes sont un grand classique d'élections anomiques, c'est-à-dire échappant à toute loi régissant habituellement les scrutins : pas d'enjeu de pouvoir, circonscription unique, mode de scrutin encourageant la dispersion, anonymat des candidatures. La sanction en est le traditionnel record des abstentions, comme des votes blancs et nuls, malgré une offre électorale élevée. Cette fois-ci encore, les résultats ont confirmé cette donnée de base : le chiffre des abstentions $(52,9 \%)$ est le plus élevé de toute l'histoire électorale de la V' République (hormis le référendum sur la Nouvelle-Calédonie de 1988); il s'est même trouvé plus d'un million d'électeurs pour faire la démarche de se rendre dans un bureau de vote pour y déposer un bulletin blanc ou nul $(5,94 \%)$. C'est aussi un record historique.

Dans ce cadre général, qu'en est-il du comportement de l'électorat du LanguedocRoussillon? Les analyses régulières des consultations publiées dans cette revue ${ }^{1}$ ont conclu à une banalisation politique d'un vote resté longtemps protestataire et atypique, celui du Midi Rouge. Cette tendance relativement récente mérite d'être vérifiée cas par cas. Elle ne gomme pas la recomposition des cultures et des traditions politiques sur un territoire où la fragmentation départementale n'est plus la donne essentielle.
Les élections européennes ont confirmé le double processus de nationalisation du vote régional et de régionalisation de particularismes politiques.

Ici, comme ailleurs, l'abstention y a battu des records : $49,12 \%$ (mais les nuls et blancs sont restés inférieurs de moitié, $3 \%$ seulement). Les écarts entre la moyenne nationale et régionale des listes n'ont pas excédé 3 $\%:+3,06 \%$ pour les "chasseurs" ; $-3,18 \%$ pour Sarkozy. Même le total des voix de l'extrême-droite (Le Pen et Mégret confondus) ne monte qu'à peine $2 \%$ de plus que dans le reste de la France. Quant à "l'effet Cohn-Bendit", il est plutôt moindre dans une région $(-0,88 \%)$ qui semble s'appliquer à ressembler aux autres.

Pour autant, les tendances singulières relevées antérieurement se confirment. La droite régionale, depuis l'exclusion de l'UDF de J. Blanc à cause de son alliance en mars 1998 avec le Front national pour conserver la Présidence du Conseil régional, est plus éclatée et plus démobilisée que jamais. Aussi bien la liste RPR-Démocratie Libérale, soutenue par J. Blanc, que celle de la nouvelle UDF, à la fois reculent encore un peu plus par rapport aux élections précédentes et font les plus mauvais résultats en moyenne régionale par rapport aux scores nationaux $(-3,18 \%$ pour Sarkozy-Madelin ; $-2,56 \%$ pour Bayrou) et ce dans un 


\section{Pôle Sud $N^{\circ} 11$}

\section{Vote CPNT aux élections européennes de 1999}

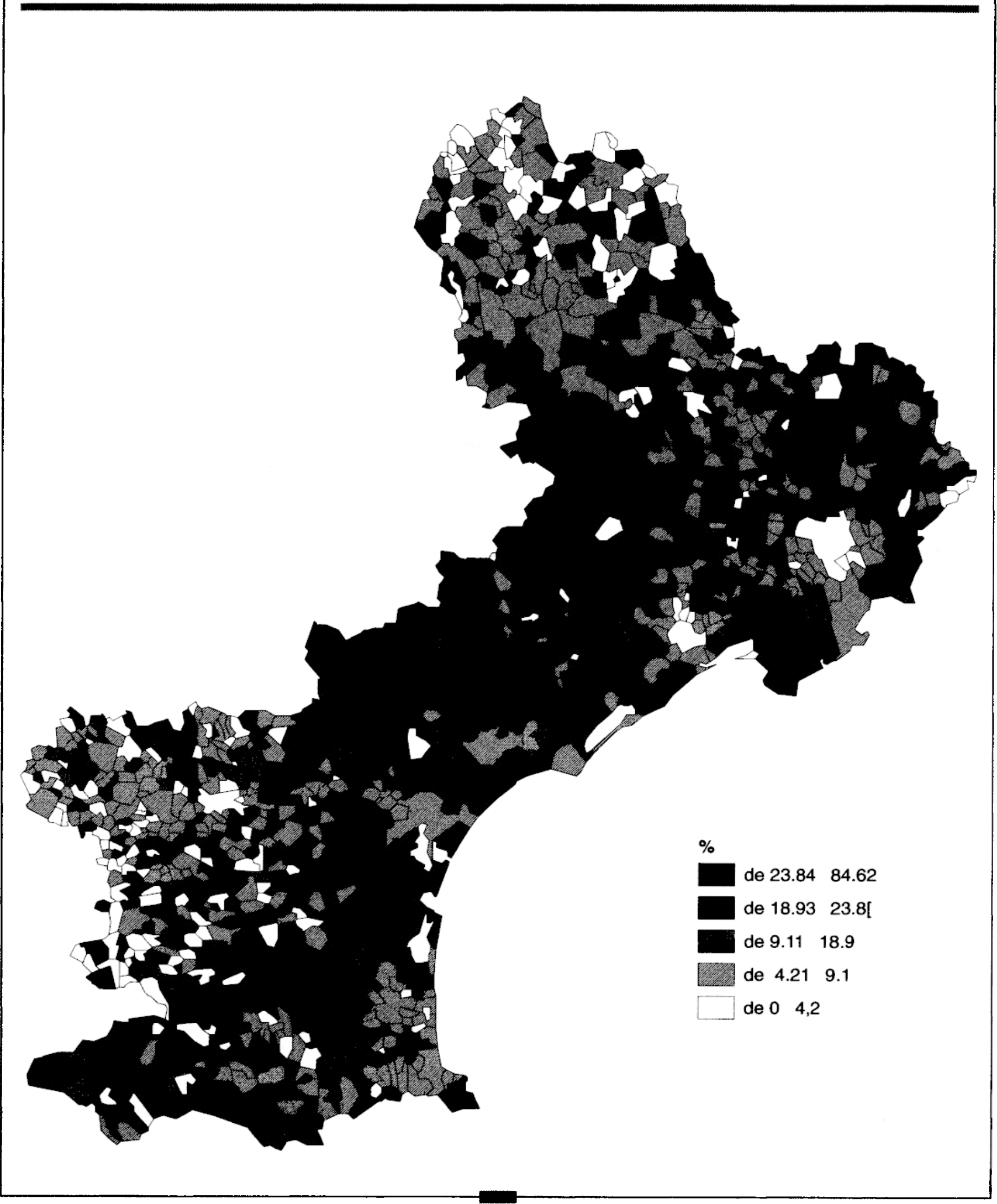




\section{Les élections européennes du 13 juin 99 en L.-R.}

contexte de stagnation et de division de l'extrême-droite. L'hypothèque de cette alliance manifestement déroutante pour les électeurs, peut être compensée dans des combinaisons localistes des états-majors pour les élections municipales (avec des listes d'union et la réduction du nombre de triangulaires) ; elle n'en reste pas moins problématique pour l'électorat.

Quant à la gauche, si sa remontée se confirme, c'est grâce à une recomposition interne qui voit l'électorat communiste régulièrement refluer. Les implantations et spécificités locales n'en sont que valorisées.

L'analyse plus fine des résultats par courants confirme cette dialectique entre nationalisation et régionalisation de l'électorat. Ainsi en va-t-il du vote en faveur de "ChassePêche-Nature-Tradition", le plus hétérogène de tous.

\section{Un vote traditionnaliste et périphérique}

Les élections sur fond d'enjeu européen cristallisent de longue date les mécontentements parmi les adeptes de la chasse. Les polémiques autour de la fixation des dates de la fermeture de la chasse ont provoqué au printemps dernier des manifestations d'envergure prouvant une détermination qui anticipait une polarisation électorale déjà perceptible dans des scrutins de liste antérieurs. Des européennes de 1989 et 1994 aux régionales de 1998 (Pôle Sud n 8, mai 1998), nous avons vu émerger une vague contestataire qui s'affermit en 1999.

A l'échelle régionale la principale zone de force est toujours centrée sur le quart nordouest de l'Hérault. Dans cette frange très rurale, la liste CPNT dépasse les $30 \%$ des suffrages exprimés dans plusieurs cantons
(Saint-Pons-de-Thomières, 34,14\% ; Olargues, 33,32\% ; La Salvetat-sur-Agout, $32,84 \%$, etc.). En peaufinant l'observation dans ce département au cinquième rang national au regard du nombre de chasseurs, on s'aperçoit que contrairement au scrutin régional la mobilisation s'accroît dans des zones littorales de chasse au gibier d'eau, visées par des directives européennes il y a peu. À Candillargues, Saint-Just, Lansargues ou Saint-Nazaire-de-Pézan, la fronde a permis aux chasseurs de recueillir en moyenne plus d'un cinquième des bulletins électoraux.

En Languedoc-Roussillon où les scores sont supérieurs à la moyenne nationale de trois points $(9,83 \%$ contre $6,77 \%)$ des espaces de force existent également du côté des Causses ( $25 \%$ dans le canton du Caylar), des Cévennes gardoises ou dans l'Aude (les chasseurs par rapport à 1994 multiplient par deux leur manne électorale dans ce département). Le Gard connaît une réelle poussée, plus mesurée dans les PyrénéesOrientales et même la Lozère, longtemps réfractaire, voit surgir un électorat favorable aux appels des défenseurs de la ruralité. Seules les cités urbaines constituent une barrière à cette influence grandissante.

Le grand enseignement du scrutin de juin 1999 réside dans le gonflement significatif des suffrages en faveur des chasseurs dans plusieurs communes périurbaines qui connaissent une croissance démographique très récente comme l'attestent les données du dernier recensement. Autour de Montpellier, si l'augmentation de la population ne date pas spécifiquement de la dernière décennie, l'extension constatée dans un périmètre de plus en plus éloigné (vers Lunel, Claret, Saint-Martin-de-Londres, 


\section{Pôle Sud $N^{\circ} 11$}

Vote RPR-PR aux élections européennes de 1999

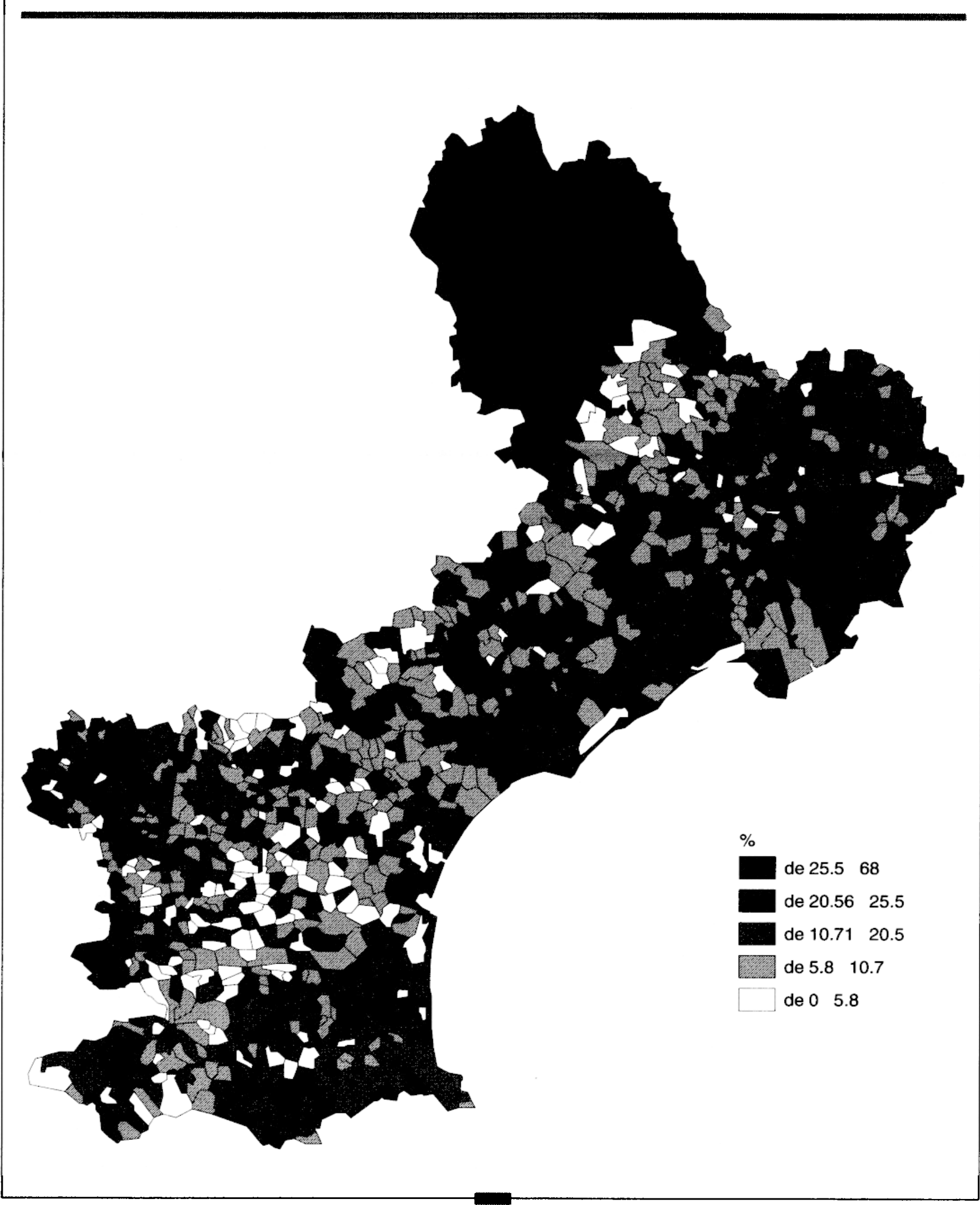




\section{Les élections européennes du 13 juin 99 en L.-R.}

\section{Evolution des scores de CPNT en Languedoc-Roussillon en \%}

\begin{tabular}{lccccc}
\hline & Aude & Gard & Hérault & Lozère & $\begin{array}{c}\text { Pyrénées- } \\
\text { Orientales }\end{array}$ \\
Européennes 1994 & 4,09 & 4,73 & 4,76 & 5,09 & 4,79 \\
Régionales 1998 & 6,28 & 3,75 & 5,67 & - & 6,54 \\
Européennes 1999 & 10,09 & 8,86 & 10,9 & 8,52 & 9,03 \\
\hline
\end{tabular}

etc.) ou dans la bande littorale (Frontignan, Mèze, etc.) grignote mécaniquement le secteur réservé à la chasse et peut provoquer des conflits avec les nouveaux arrivants attirés par la fibre écologique et peu disposés à supporter le comportement des traqueurs de gibier. Au niveau du canton, la liste CPNT obtient $16,93 \%$ à Saint-Martin-deLondres, $15,7 \%$ des voix à Claret, $14,6 \%$ à Frontignan, 14,37\% à Lunel...

On peut voir poindre ici un affrontement entre deux logiques. Une qui émane des "néo", que l'on pourrait appeler "consommatrice". Elle souhaite encourager l'éclosion d'un espace de loisir suffisant (aménagement de sentiers de randonnées, de "parcours santé", de pistes destinées aux VTT ou aux motards, etc.) qui souvent heurte les habitudes des chasseurs locaux. Ceux-ci défendent une logique plus "traditionnelle". Ils entendent préserver, coûte que coûte, une passion qui rythme leur vie depuis des générations. Le territoire de chasse doit rester un espace immuable dans une société où tout va très vite et qui n'a pas toujours digéré l'effondrement d'une viticulture de masse et tout ce qu'elle incarnait comme lieu de socialisation au sein du "Midi rouge". Après avoir initié la politique d'arrachage, la "technocratie européenne" viserait désormais les irréductibles chasseurs regroupés derrière quelques notables des Fédérations départementales (Alain Esclopé, le conseiller régional catalan, élu en sixième position; Jean
Benoît à la tête des revendications dans l'Hérault, etc.) bien organisés pour orchestrer une mobilisation qu'ils espèrent durable.

\section{Une droite éclatée et désorientée}

Depuis l'élection de plusieurs présidents de région, dont Jacques Blanc, avec l'apport des voix de l'extrême-droite un séisme politique a ravagé les formations de la droite parlementaire qui n'en finissent pas de chercher une solution pour éviter un éclatement définitif. Les tentatives faites jusqu'aux élections européennes n'ont rien donné de concret. Aussi la brève campagne, éclipsée par la guerre dans l'ex-Yougoslavie, a été placée sous le signe de la division. Le RPR, abandonné par Philippe Séguin, allié à DL se voit concurrencer par la "nouvelle UDF" et le tandem Pasqua-Villiers, réuni sous la bannière d'un Rassemblement pour la France hostile à l'Europe en construction.

Les notabilités de la droite régionale écartelées entre une fidélité à Jacques Blanc, une attitude de neutralité bienveillante ou plus rarement d'hostilité ne figurent guère à des positions éligibles sur les listes en course. Déjà très touchées lors des dernières législatives (seul Jacques Blanc siège encore au PalaisBourbon), aucune ne représentera la région à l'assemblée de Strasbourg. Le maire de Perpignan, Jean-Paul Alduy, est le mieux placé si l'on prend en compte les trois fractions 


\section{Pôle Sud $N^{\circ} 11$}

concurrentes; il se trouve seulement à la $14^{\circ}$ place. Son homologue du Canet-en-Roussillon, Arlette Franco, qui participe à la majorité régionale, n'apparaît qu'en vingt-septième position. Un examen attentif des listes permet d'apercevoir éparpillé dans les tréfonds du classement quelques militants reconnus (Bruno Barthez, conseiller municipal d'opposition à Montpellier, en $70^{\mathrm{c}}$ position défend les couleurs de l'UDF et l'ultralibéral Jean Giménez figure une place plus loin sur celle dirigée par Nicolas Sarkozy et Alain Madelin) ou d'obscurs sans-grade (le premier représentant régional inscrit sur la liste de Charles Pasqua pointe au soixantième rang, etc.). Parmi toutes les hypothèses envisageables pour expliquer la faiblesse de la représentation des candidatures régionales, on peut avancer le réel discrédit qui entoure un personnel incapable depuis plusieurs années de bâtir des structures partisanes solides aux yeux des directions nationales, contesté sur sa droite par les poussées frontistes, dépourvu de suffisamment de fantassins notabiliaires à l'échelle cantonale.

Une vague de fond a laminé le rassemblement gaulliste soudé à la branche héritière du PR au profit de la gauche plurielle et de la mouvance Pasqua-Villiers, laquelle arrive en tête de toutes les listes d'opposition hormis en Lozère. L'influence discrète de Jacques Blanc, réintégré en catimini dans le groupe parlementaire DL en août 1998 après sa mise au ban de la droite modérée, subsiste. Toutefois si l'on compare avec précaution, compte tenu des mutations du paysage politique national, le résultat avec celui des dernières élections européennes dominées par une union bien lointaine, la baisse est flagrante. Dominique Baudis réalisait près de $40 \%$ des suffrages alors que l'addition des voix de toutes les composantes, à l'exception du RPF, ne dépassait pas les $30 \%$. Dans l'Hérault, le secrétaire départemental du RPR, Stéphan Rossignol n'hésite pas à balayer la "langue de bois" pour avouer qu'il s'agit d'un "mauvais résultat" (Midi Libre, 15 juin 1999). Avec une perte sèche de 7 points par rapport à 1994 dans le département $(8,95 \%$ pour RPR-DL et $6,31 \%$ pour l'UDF contre 21,9 $\%$ pour Baudis), la droite subit un nouveau revers encore plus accentué dans l'Aude (-10 points) alors qu'il est relativement limité ailleurs ( -5 points dans le Gard et -2 points dans les Pyrénées-Orientales). Les cartes ne laissent guère entrevoir d'îlots de résistance. On note simplement un léger "effet Alduy" cantonné à quelques secteurs autour de Perpignan (mais bien réel à Perpignan ville où Bayrou dépasse les $14 \%$ ) et dans la bordure sud de cette terre catalane.

Le scrutin a donc surtout été marqué par la percée de Charles Pasqua, en deuxième position dans les cinq départements. La faconde

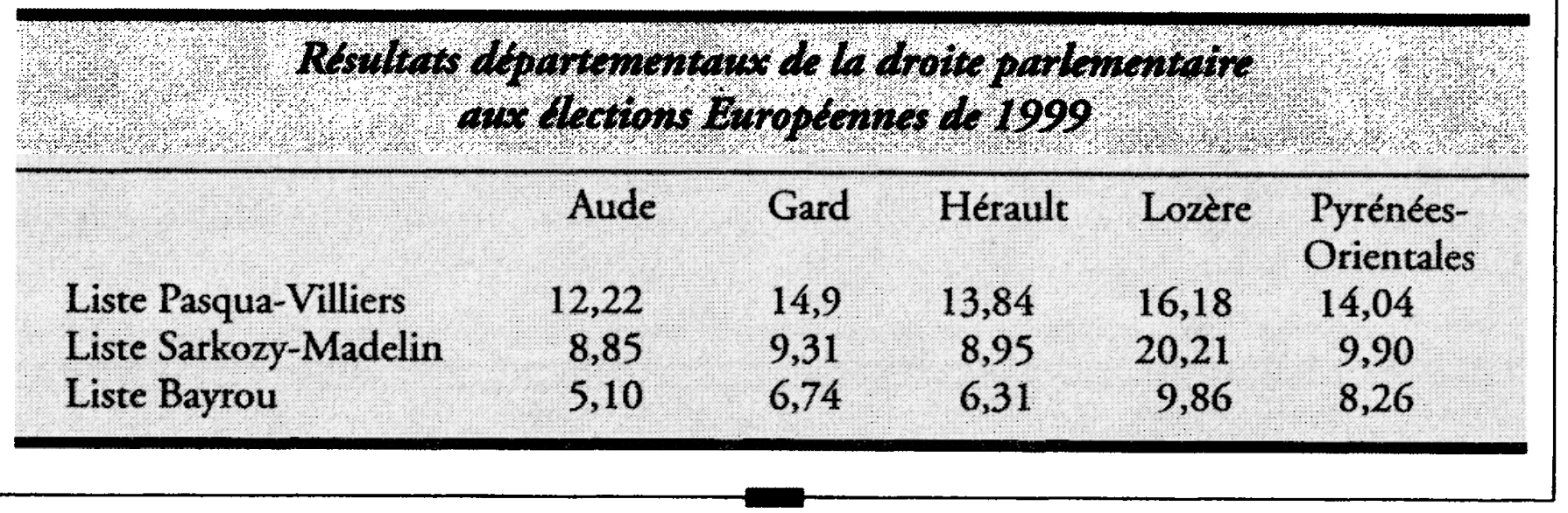


Les élections européennes du 13 juin 99 en L.-R.

\begin{tabular}{|c|c|c|c|c|c|}
\hline 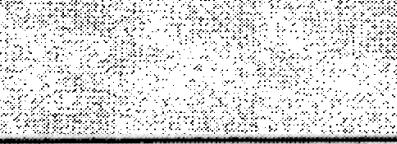 & $\begin{array}{l}\text { indes: } \\
\mathrm{cm} \operatorname{lm}\end{array}$ & 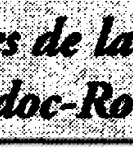 & low & & \\
\hline & Aude & Gard & Hérault & Lozère & $\begin{array}{l}\text { Pyrénées- } \\
\text { Orientales }\end{array}$ \\
\hline Européennes 1994 & 8,62 & 10,43 & 10,81 & 12,06 & 11,91 \\
\hline Européennes 1999 & 12,22 & 14,90 & 13,84 & 16,18 & 14,04 \\
\hline
\end{tabular}

Vote RPF aux élections européennes de 1999

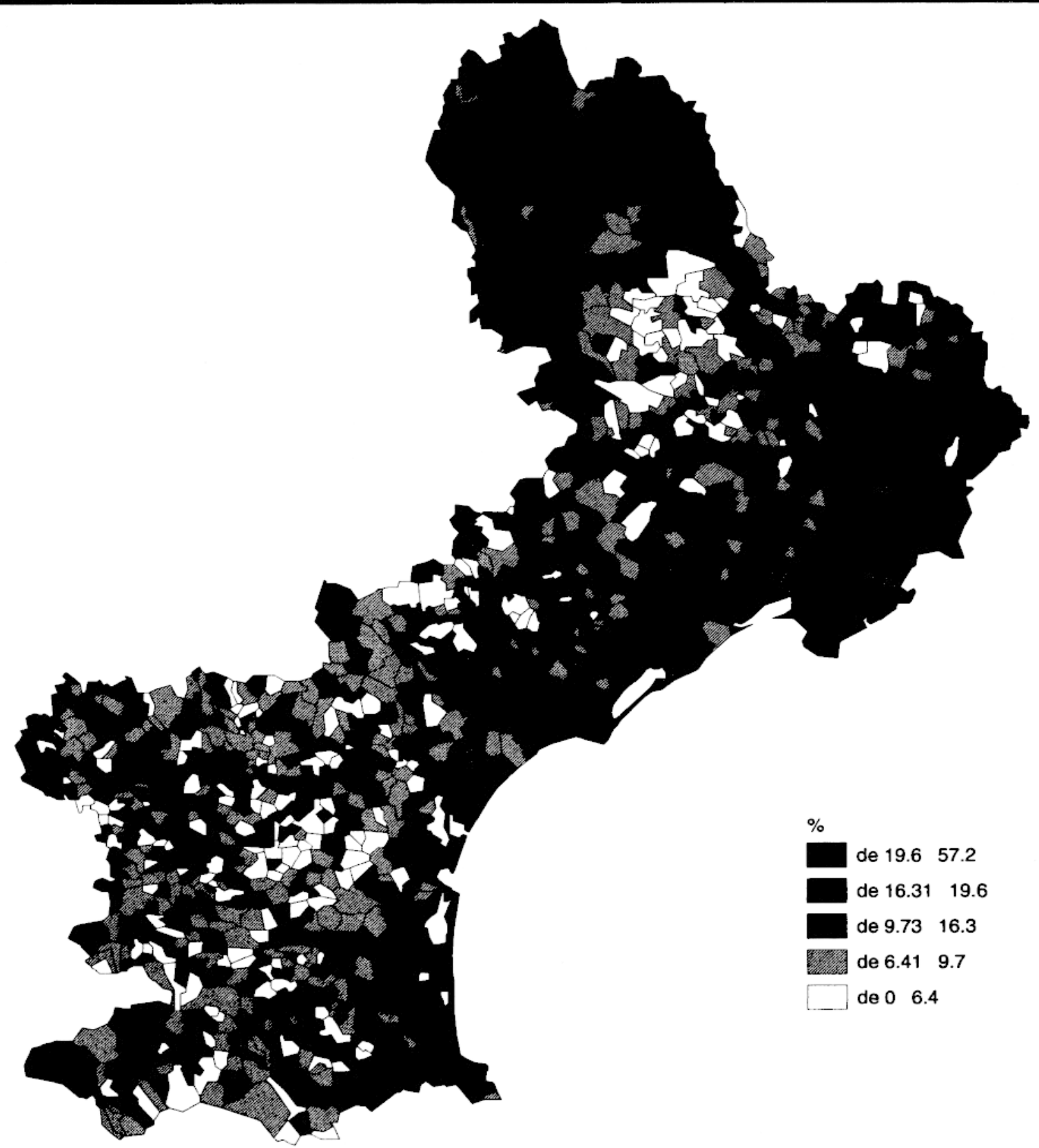




\section{Pôle Sud $N^{\circ} 11$}

de l'ancien dirigeant du RPR dope le réservoir de voix de Philippe de Villiers. Lalliance de ces deux ténors hostiles à l'Europe de Maastricht permet au Rassemblement pour la France de progresser globalement de 4 points en comparaison avec 1994 et d'absorber un électorat de droite à la fois désorienté par les facéties des leaders nationaux et réticent devant la construction européenne.

Dans la plupart des villes languedociennes, le RPF se présente comme la principale force d'opposition alors même qu'un maigre contingent d'élus locaux s'était risqué à soutenir ce tandem de contestataires à l'exception notable de Raymond Chésa qui dans sa ville de Carcassonne récolte $15,45 \%$ des voix. Ailleurs (comme à Béziers par exemple) les leaders reçoivent un désaveu dont il est aujourd'hui bien difficile de déceler la portée. Soit Charles Pasqua arrive à capitaliser par l'intermédiaire d'un maillage organisationnel ce premier pas et à entraîner dans son sillage des notables déçus ou des militants capables d'impulser un souffle nouveau; soit il ne s'agira que d'une simple flambée électorale sans lendemain.

L'état de la droite en Languedoc-Roussillon, au sortir de ces européennes est à l'unisson de sa situation dans le reste de la France. Mais il est manifestement obéré par le poids de la stratégie imposée depuis 1998 et du haut de la région à l'ensemble des formations. L'alliance passée alors avec l'extrême-droite continue à isoler les représentants locaux de la droite républicaine et à limiter leurs marges de manceuvre nationale. Cela peut devenir une ressource pour les élections municipales. Mais la crise du Front National n'a pas modifié la donne politique de la droite comme nous allons l'observer maintenant.

\section{Evolution departementale des scores du Front National}

en Languedoc-Roussillon (en \% des suffrages exprims)

\begin{tabular}{|c|c|c|c|c|c|}
\hline & Aude & Gard & Hérault & Lozère & Pyrénées $\mathbf{O}$. \\
\hline Européennes 1984 & 8,5 & 12,7 & 15,3 & 6,7 & 15,9 \\
\hline Régionales 1986 & 6,1 & 14,8 & 14,1 & 5,1 & 17,8 \\
\hline Présidentielles 1988 & 13,7 & 20,6 & 19,9 & 11,6 & 20,5 \\
\hline Européennes 1989 & 10,0 & 14,8 & 15,9 & 8,0 & 18,7 \\
\hline Régionales 1992 & 11,6 & 18,2 & 18,3 & 5,9 & 22,6 \\
\hline Législatives 1993 & 11,8 & 17,8 & 14,9 & 5,6 & 18,3 \\
\hline Européennes 1994 & 8,8 & 13,8 & 13,3 & 6,2 & 14,8 \\
\hline Présidentielle 1995 & 13,6 & 20,3 & 19 & 9,6 & 19,5 \\
\hline Législatives 1997 & 14,4 & 21,3 & 19,3 & 8,3 & 21,2 \\
\hline Régionales 1998 & 13,2 & 21,9 & 17,3 & 5,7 & 17,9 \\
\hline \multicolumn{6}{|l|}{ Européennes 1999} \\
\hline Ensemble & 8,28 & 13,36 & 10,83 & 5,42 & 11,62 \\
\hline dont $F N$ & $5,277,61$ & $6,093,17$ & 7,18 & & \\
\hline$M N$ & $3,015,75$ & $4,742,25$ & 4,44 & & \\
\hline
\end{tabular}




\section{Les élections européennes du 13 juin 99 en L.-R.}

\section{Une extrême-droite stagnante et dispersée}

Les élections européennes sont, depuis l'émergence du Front national dans l'espace politique français, celles qui sont en termes de suffrages les moins favorables à l'extrêmedroite. Comme on peut le voir sur le tableau suivant, cette tendance est régulièrement affirmée depuis 1984. Le score comparativement faible des formations qui sont issues de cette mouvance pour les élections européennes de 1999 doit cependant être analysé à la lumière de la lutte interne au Front national en 1998, qui a débouché en 1999 à la scission en deux forces, Front national (J.-M. Le Pen) et Mouvement national (B. Megret) se présentant séparément aux urnes. Les effets de cet affrontement interne à l'extrêmedroite sont particulièrement intéressants à analyser en Languedoc-Roussillon, pour au moins trois raisons. La première réside dans le fait que cette région, qui comptait un député européen sortant (J.-C. Martinez, hiérarque de l'actuel Front national, réélu), est aussi celle de l'un des principaux acteurs de la contestation, puis de la fondation du Mouvement national et de la coalition régionale UDF-RPR-FN-MN (S. Martinez). L'implantation languedocienne du Front national, très forte dans certaines zones de la région (Pôle Sud, 1998), va donc de pair avec un statut de foyer spécifique de la compétition et du leadership. Il était intéressant d'analyser la stabilité et les transformations éventuelles de cette implantation territoriale. L'extrême-droite y occupe ensuite une place particulière au travers de son intégration à la majorité qui gouverne le Conseil régional. Les mouvements qui affectent à la fois le vote extrême en général et le rapport de force entre ses deux expressions politiques y sont donc particulièrement importants à suivre du point de vue du système politique régional en général.

Nous avons choisi de centrer cette brève analyse sur la comparaison de ce scrutin avec les précédentes élections régionales. S'il s'agit d'élections profondément distinctes du point de vue de leur objet, de leur représentation et des taux de participation, elles n'en ont pas moins deux points communs au moins : le mode proportionnel et un statut d'élection intermédiaire qui permettent, à un an d'intervalle, de tirer quelques solides enseignements quant à l'évolution du vote extrême. Nous allons d'abord nous intéresser à l'évolution des tendances globales de ce vote, en analysant les résultats des deux listes FN et MN cumulées. Nous examinerons ensuite le rapport de forces tel qu'il ressort de la première consultation électorale postérieure à la scission entre deux formations.

\section{Un repli mesuré du vote extrême}

A l'image des résultats nationaux, le repli du vote d'extrême-droite est le premier enseignement de ce scrutin. Le chiffre relativement élevé d'abstentions lors de ces élections, associé à ce reflux global, permet d'avancer l'hypothèse que les zones où l'influence des deux formations demeure (ensemble) plus élevée que la moyenne sont celles d'un ancrage plus conséquent, plus stable de ce comportement électoral. Cette hypothèse est renforcée par le fait que l'offre était plus conséquente encore que pour les dernières élections régionales; la possibilité de changer de vote (quels qu'en soient les motifs) plus ouverte encore. C'est en fonction de ces éléments qu'il faut évaluer globalement un vote $\mathrm{FN}-\mathrm{MN}$ qui se situe d̀ un 


\section{Pôle Sud $N^{\circ} 11$}

niveau certes inférieur aux précédentes consultations, mais parfois de fort peu (moins de $1 \%$ d'écart par rapport aux élections de 1994 dans l'Aude, le Gard et la Lozère, autour de 3\% dans l'Hérault et le Pyrénées-Orientales).

Lorsque l'on cumule les résultats FN et $M N$, on observe tout d'abord un repli assez homogène dans les zones que l'on peut considérer comme les bastions du vote d'extrêmedroite (le sud gardois, les zones urbaines en général, et notamment celles qui sont frappées par des phénomènes de récession industrielle, le littoral). Ces zones connaissent toujours des scores supérieurs à la moyenne régionale, mais revus à la baisse dans des proportions nettes, sans toutefois manifester un effondrement. On notera que certaines villes font exception à un tassement modéré. A Castelnaudary (Aude), il fait mieux que résister à la baisse. C'est toutefois la seule exception urbaine véritable. A Nîmes au contraire, le vote FN-MN s'écroule - il perd plus de dix points, avec un score de $14,27 \%$, soit $6,61 \%$ pour la liste Mégret et 7,66\% pour celle de J.M. Le Pen faisant vraisemblablement les frais du succès relatif (15,7\%) de la liste Pasqua-De Villiers).

En dehors de cette exception nîmoise, les zones de forte baisse, de même que les zones de stabilité (voire de hausse) du vote d'extrême-droite sont toutes des zones rurales de la région, où le vote FN était peu représenté. L'exemple de la Lozère est à cet égard caractéristique : le vote FN n'y était supérieur à la moyenne régionale que dans six communes. On en compte désormais 22 dans ce cas : la baisse de l'étiage régional fait ainsi apparaittre des zones d'ancrage lozériennes, où les changements conjoncturels de comportements sont sans doute plus rares. D'ailleurs, les communes rurales où ce vote chute ne sont pas celles où les suffrages se reportent sur les chasseurs. Ce sont bien au contraire des communes qui "reviennent" à un vote en faveur de la droite républicaine.

On fait un constat très différent dans les quatre autres départements, où la perte de voix FN-MN coïncide avec une croissance du vote "chasseurs" (CPNT) assez remarquable. Celui-ci démarque par ailleurs un territoire de chasse et de pêche (les hauts-cantons de l'Hérault, les communes de bord d'étang, telles que Gruissan par exemple).

Ces premiers constats (baisse homogène sur les bastions, maintien/progression ou effondrement en zone rurale) doivent toutefois tenir compte du fait que les variations du vote rural concernent des chiffres souvent très faibles. Par exemple, une partie des communes comprises dans le périmètre du Pays Cathare voient certes le vote FN-MN progresser, mais ce sont toutes des communes de très faible effectif électoral, et ces scores demeurent par ailleurs inférieurs à la moyenne régionale, elle-même en baisse. Ceci incite au contraire à s'intéresser aux zones qui, tout en ayant vu chuter l'influence du FN-MN cumulée, sont celles où les scores demeurent supérieurs à la moyenne régionale.

\section{La stabilité de structure de l'implantation électorale}

Les trois espaces où le FN et le $\mathrm{MN}$ jouent leur influence électorale restent les mêmes que celles mises en évidence à l'occasion des élections régionales de 1998.

Le Gard, celui qui avait manifesté la plus grande ampleur de pénétration du vote FN, est le seul à conserver des scores très élevés (parfois encore supérieurs à 20\%). Tout le Sud (avec des communes héraultaises limitrophes) et une notable partie de l'Ouest du département demeurent terre d'élection 


\section{Les élections européennes du 13 juin 99 en L.-R.}

pour l'extrême-droite, tandis que le Nord cévenol continue de résister à son implantation, sauf dans les zones de reconversion industrielle. C'est sans doute ici que se joue, plus que dans toute autre zone, l'avenir politique de l'extrême-droite, et le rapport de forces entre les deux mouvances.

Le littoral est la seconde zone de maintien d'un vote FN-MN supérieur à la moyenne. L'exception à la règle, en 1998, était la commune de Collioure. C'est désormais le cas de Gruissan, Candillargues et Lansargues où le vote Chasseur a manifestement rallié une partie des électeurs FN à sa cause. Nous ne reviendrons pas ici sur les hypothèses explicatives d'un tel constat (présence d'un électorat âgé, habitat dans des zones à variation saisonnière très forte, impliquant des réseaux de sociabilité et un rapport spécifique à l'altérité), qui supposeraient d'être étayées par des études qualitatives.

Les zones urbaines et péri-urbaines constituent la troisième terre d'influence FN-MN. Au-delà des villes, pour lesquelles chaque élection fait disparaître son lot d'exception (ici Castelnaudary ou Alès), c'est la stabilité de cette influence dans les zones péri-urbaines qui mérite d'être remarquée. Nous avions pointé une certaine coïncidence entre le vote $\mathrm{FN}$ et le développement d'une urbanisation spécifique en zone anciennement rurales. C'était en particulier le cas du péri-urbain entourant les villes principales et de certains couloirs d'urbanisation correspondant à des axes interurbains (Montpellier-Sommières, Montpellier-Quissac par exemple). Cette caractérisation se précise. On peut aujourd'hui constater que ce phénomène (tout en tenant compte du tassement global du vote) s'étend à la presque totalité des communes qui ont développé des programmes d'urba- nisation active ciblés sur les revenus moyens. Le Languedoc de ces nouveaux petits propriétaires mériterait sans doute une analyse plus fine qu'un simple constat de statistique électorale. Notons toutefois qu'il concerne un nombre d'électeurs en forte augmentation, puisqu'il coïncide avec les communes où la croissance démographique a atteint des records lors du dernier recensement.

Il reste à identifier le protagoniste (FN ou $\mathrm{MN})$ qui tire le moins mal son épingle d'un jeu électoral globalement défavorable au vote extrémiste.

Comment la scission se traduit-elle dans les urnes régionales ? Du point de vue spatial, les cartes découpées par les scores du $\mathrm{FN}$ et du MN sont étonnamment proches : plus de soixante-dix pour cent des communes de la région se retrouvent dans les mêmes échelles d'influence. En d'autre termes, les cartes dessinées par la distribution des deux courants, le FN de Jean-Marie le Pen et le MN de Bruno Megret, sont des décalques l'une de l'autre.

\section{L'absence de partage du territoire}

Sur les 83 communes de la région dans lesquelles le cumul FN+MN dépasse les $16 \%$ des suffrages, 18 sont des communes où le $\mathrm{FN}$ et le MN réalisent leurs meilleurs scores, 20 sont des communes bastion du FN, 21 des communes bastion du MN. Dans cet ensemble rapproché, le sud gardois reste le bastion indéniable de l'extrême-droite, et notamment l'ensemble contiguë de 16 communes entourant Nîmes, depuis Vauvert, au sud-ouest, à Sernhac, à l'Est. Deux autres zones traditionnelles sont elles aussi partagées par les deux courants : les grandes communes urbaines de la région, et la large bande littorale et péri-urbaine décrite précédemment. La 


\section{Pôle Sud $N^{\circ} 11$}

Vote FN et MN aux élections européennes de 1999

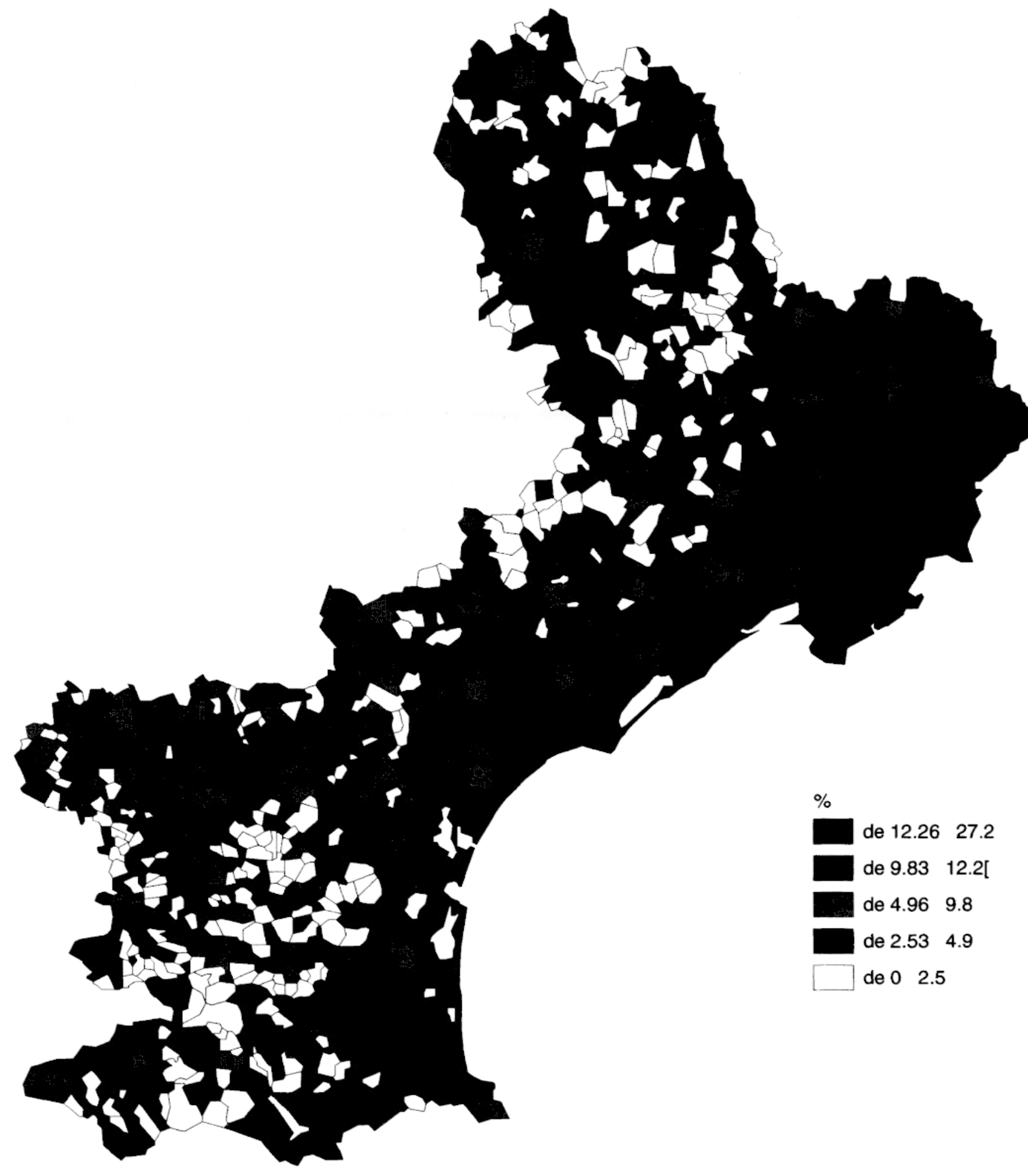




\section{Les élections européennes du 13 juin 99 en L.-R.}

scission ne se traduit pas par un Yalta territorial. Les deux courants marchent sur les mêmes terres, se partagent un potentiel construit précédemment, potentiel affaibli néanmoins par leur compétition. Seul point de discordance, les petites communes rurales qui donnent depuis une dizaine d'années une part importante de leurs voix au FN ont, le plus souvent, choisi leur camp. Le FN connaît ainsi de fortes pointes au sud de la région, dans les corbières et les vallées de l'Aude, tandis que le $M N$ poursuit une progression au nord, en Lozère notamment. Si ces différences restent très marginales, elles ouvrent la voie à deux hypothèses de structuration des électorats.

Tout d'abord, le vote national-populiste reste un vote de diffusion de proximité. C'est dans les petites communes d'implantation traditionnelles, dans lesquelles le FN constituait un fort courant en valeur relative (+ de $15 \%$ ), mais faible en valeur réelle (une dizaine d'électeurs), que le basculement vers l'un ou l'autre des deux courants est le plus net, et le plus visible. La présence d'un relais de l'un ou l'autre des courants, agissant sur de faibles populations d'électeurs, expliquent ces basculements désordonnés dans les petites unités rurales.

Mais au-delà de ces micros explications, une tendance se dégage néanmoins quant aux vecteurs de diffusion des deux courants, le MN semblant mieux se développer dans les territoires ancrés à droite. On retrouve cette tendance dans l'évolution des votes lozériens, mais également dans la distribution spatiale sud-gardoise.

L'étude de cette dernière zone offre un autre enseignement, celui de l'échec relatif du $\mathrm{MN}$. Zone de prédilection de son développement, autour d'un de ses leaders nationaux, Serge Martinez, elle n'est pourtant pas un bas- tion exclusif du MN. Plus, celui-ci n'est pas arrivé à y dépasser son rival frontiste. Rares sont en effet les zones où les mégretistes ont atteint cet objectif. Dans les grandes zones d'implantation de l'extrême-droite régionale, seuls les électorats frontistes du quart NordOuest du Gard, ainsi qu'une partie des agglomérations montpelliéraine et carcassonnaise ont majoritairement basculé du côté de Bruno Mégret. Si le $\mathrm{MN}$ semble mieux réussir dans les zones montagneuses et rurales, il perd indéniablement son combat dans le grand sud gardois, mais aussi dans tous les ensembles urbains (Béziers, Perpignan, Narbonne) et sur le littoral. L'existence d'un meilleur rapport de forces régional avec le FN ne saurait masquer le fait qu'il ne passe pas, en région, la barre des $5 \%$, ce malgré un mauvais score du FN, et l'échec continu de l'implantation des deux leaders lepénistes.

\section{Une extrême-droite sans véritables bastions}

Dernier enseignement, l'extrême-droite régionale se retrouve privée de véritables bastions municipaux à l'issue de ce scrutin. Alain Jamet dirige à Montpellier un FN tout juste au-dessus de la barre des $5 \%(5,57)$. Jean-Claude Martinez essuie un nouvel échec à Sète, avec $7,01 \%$ des voix. Des bourgs-centres comme Lunel, longtemps considérés comme des bastions en formation, subissent de plein fouet la concurrence des deux courants, qui s'établissent chacun à 8,5\%. La division quasi parfaite des voix (avec un léger avantage au FN) dans les grands centres urbains diminue ainsi considérablement le potentiel d'implantation et le pouvoir de nuisance de l'extrême-droite.

En effet, cet affaiblissement par la division ne sera pas sans conséquence sur les autres 


\section{Pôle Sud $N^{\circ} 11$}

\section{Répartition territoriale du FN et du MN}

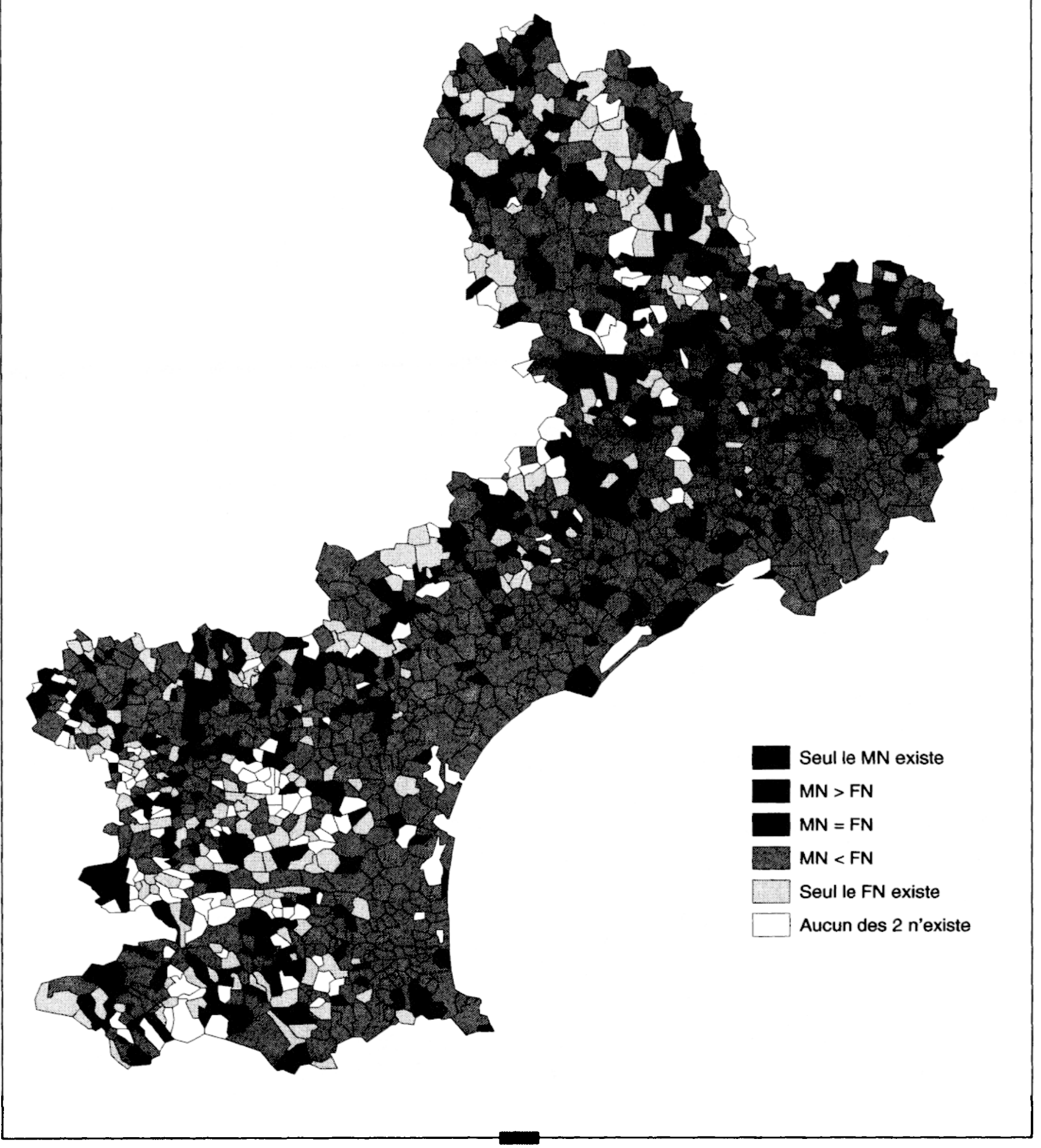




\section{Les élections européennes du 13 juin 99 en $L .-R$.}

élections et les prochaines configurations électorales. Vainqueur par $\mathrm{KO}$ aux dernières élections législatives et cantonales, la gauche régionale devrait se voir privée de triangulaires qui ont permis l'élection de plusieurs de ses députés actuels.

On aurait pourtant tort de considérer que le scrutin européen sonne le glas de l'ancrage politique de l'extrême-droite en LanguedocRoussillon. Si son tassement électoral est manifeste, les deux formations qui s'en disputent la représentation continuent d'être présentes dans les mêmes zones qui avaient fait son succès. Cette stabilisation, même revue à la baisse, interdit d'écarter ses élus et leaders de la donne politique languedocienne des prochaines élections municipales et législatives notamment. Le rapport de forces entre les deux courants doit être, comme au niveau national, analysé avec prudence compte tenu de la proximité de date entre la crise fatale à l'unité du mouvement et la consultation ellemême. Il constitue enfin à n'en pas douter un facteur de complexification, si cela était nécessaire, des jeux politiques au sein de la majorité du Conseil régional.

\section{Une gauche recomposée}

A l'occasion de notre analyse des élections régionales de 1998, nous risquions l'hypothèse d'un changement de cycle électoral pour l'ensemble de la gauche. La période19771993 avait été celle de l'épuisement du cycle long des implantations territoriales et culturelles et du cycle court du renouvellement du courant socialiste à Epinay. La régression du PS dans l'Aude, le biterrois, le Roussillon comme celle du communisme municipal à Sète, Nîmes ou Alès en étaient les signes les plus connus.
La série des élections successives depuis 1995 révèle l'ouverture d'une autre période faite de reconquêtes de bastions traditionnels appuyées sur un changement sociologique et politique. Les résultats des élections européennes de 1999 le confirment. C'est particulièrement vrai en comparant ces derniers avec ceux des élections européennes de 1994, exercice on le sait indispensable en l'occurrence.

La différence n'est pas dans le nombre de suffrages recueillis régionalement par la gauche plurielle en 1999 et $1994: 39,46 \%$ contre $41,98 \%$. Encore faut-il préciser que le dernier pourcentage additionne aux chiffres de la gauche traditionnelle non seulement ceux des Verts et Génération Ecologie de l'époque mais aussi ceux de Bernard Tapie (dont les 13,55\% excédaient le vote radical et les clivages habituels). En Languedoc-Roussillon, la gauche oscille désormais autour de $40 \%$ dans toutes les élections nationales comme locales et quel que soit le mode de scrutin. C'est, en flux, une dizaine de point de plus qu'à la fin du cycle antérieur. Mais un des aspects intéressants de cette donnée est sa structuration politique interne.

Le parti Socialiste confirme son rôle de pôle stabilisateur. Le différentiel entre son score régional et national n'est pas significatif: $-0,18 \%$ en $1994 ;+0,009 \%$ en 1999 et reste en-deçà des résultats de 1989 quand la liste Fabius y faisait $24,52 \%$ (+ 1,46\% que dans la France entière). Il est très exactement dans la moyenne nationale $(22,03 \%)$, ce qui confirme la fin de son exception méridionale. Quand le centre va, la région suit et inversement. Mais la tradition vitupérante et protestataire semble être devenue électoralement contre productive. D'ailleurs, ce sont les fédérations les plus légitimistes comme celles de l'Aude (deuxième département de France à avoir le plus voté socialiste derrière l'Ariège) 


\section{Pôle Sud $N^{\circ} 11$}

Votes PS, Verts et PCF aux élections européennes de 1999

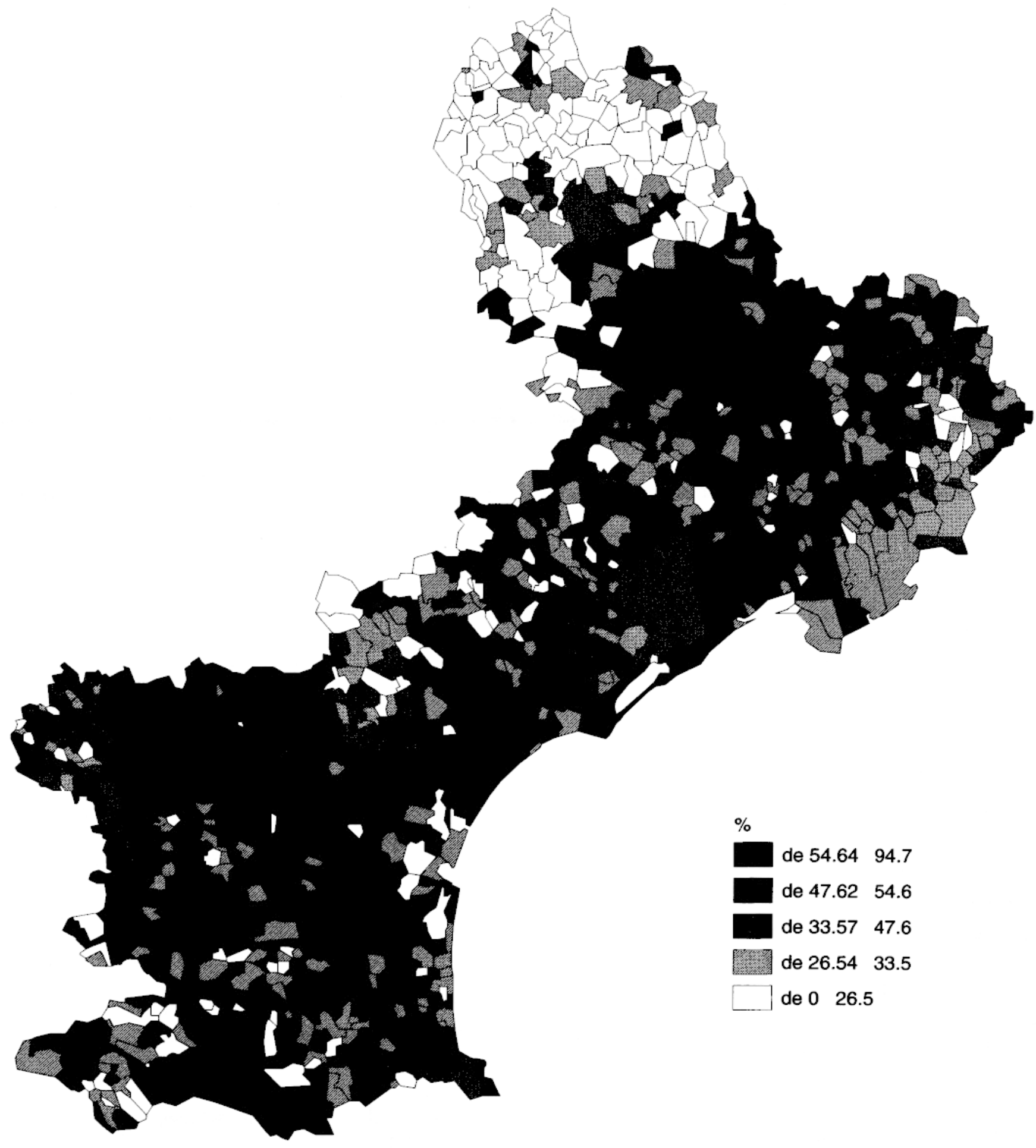




\section{Les élections européennes du 13 juin 99 en L.-R.}

qui, en retrouvant des scores très élevés, assurent le maintien de cette moyennisation. Si François Hollande fait $7,35 \%$ de mieux que Michel Rocard en 1994, c'est en effet grâce à l'Aude $(31,1 \%)$, soit une progression sur 1994 de près de 9 points, le record régional. Il est vrai que déjà à l'époque cette fédération très fabiusienne avait donné $22,15 \%$ à Rocard, un record là, national. Le phénomène est très proche dans les Pyrénées-Orientales : $+8,75 \%$ en 1999 (21,37\%). C'est curieusement dans l'Hérault que la progression est la moindre $(+6,8 \%$ et un $21,14 \%$ inférieur à la moyenne nationale) alors que la fédération y est réputée très proche de la direction nationale du parti. On ne saurait s'en tenir bien sûr à cette variable organisationnelle pour expliquer les différences d'un département à l'autre. Et la concentration urbaine de couches nouvelles et récemment installées autour de Montpellier est un facteur social et démographique d'importance. Mais l'offre électorale sur la longue durée est sans doute pour quelque chose dans ces différences. Ainsi le renouvellement général et le rajeunissement des candidats aux législatives de 1997 (dans les circonscriptions de Carcassonne, Narbonne, Perpignan, Rivesaltes) a pu jouer un rôle. De même que la composition des listes dans ces départements pour les régionales. Mais il semble bien que le degré d'adhésion à la politique gouvernementale ainsi qu'à l'appareil du parti soit un facteur supplémentaire de réussite. En tout cas, la carte du vote socialiste issue de ce dernier scrutin montre une forte concentration dans le sud-ouest de la région dans le triangle de CastelnaudaryNarbonne-Banyuls, un essaimage diffus dans le triangle Montpellier-Nîmes-Mende, et un axe de bonne résistance dans la vallée de l'Hérault (du haut-lodévois à Pézenas-Florensac). La frange littorale, hormis à son extrême- sud (Argelès-cerbère), dans le narbonnais (Gruissan-Port-La-Nouvelle) et à Frontignan, est terre de mission. Tout se passe comme si le poids du montpelliérain qui était devenu si important dans le cycle précédent perdait régionalement de sa centralité dans le vote socialiste et surtout dans les élections générales (cela est moins vrai dans les élections locales). On peut y voir une recomposition sociologique de cet électorat, au sortir de la grande transformation viticole : il est plus homogène et principalement dans les couches moyennes urbaines, celles-ci se distribuant dans le réseau des petites villes particulièrement dense, actif et ancien dans cette région. Cette dimension constitue une différence capitale avec les autres composantes électorales de la gauche plurielle et d'abord le parti communiste.

Le vote Hue, s'il se situe à $+1,82 \%$ $(8,6 \%)$ de la moyenne nationale, traduit néanmoins une nouvelle décroissance par rapport à 1994:-1,22\%. Ce mouvement se traduit par une érosion continue des bastions à de rares exceptions près comme Sète. Dans le Gard par exemple, le PC perd plus de deux points par rapport aux européennes de 1994 et près de cinq points par rapport aux régionales de 1998. À Nîmes comme à Alès, il est largement devancé par le PS (qui fait le double de ses voix dans la première ville et deux points de plus dans la seconde). Ailleurs le tassement est général : aussi bien dans l'Aude $(-1,33 \%)$ que dans les Pyrénées-Orientales $(-1 \%)$. Même à Sète où il fait exactement le même score qu'en 1994 (16,6\%), il ne devance le PS que de 0,37\% alors qu'il était à huit points devant cinq ans plus tôt. De ce fait, l'implantation communiste devient de plus en plus fragmentaire et éclatée dans l'espace régional. Les places fortes locales risquent donc de gagner en 


\section{Pôle Sud $N^{\circ} 11$}

autonomie par rapport aux structures fédérales. Le communisme municipal s'en trouvera renforcé de même que les logiques gagnantes, c'est-à-dire, depuis quatre ans celles de l'alliance privilégié avec le PS. Aux municipales de 1995 comme aux législatives, c'est là où cette logique avait été défendue par le $P C$ et qu'il en avait retiré le plus de fruits électoraux que ce soit à La Grand Combe, Nîmes ou Sète ou dans la $1^{\text {re }}$ circonscription des Pyrénées-Orientales. Le problème est que cette stratégie n'est au mieux qu'un frein mis à un plus grand reflux. Là où le PS est très fort comme à Montpellier, le PC y est déjà devancé par l'extrême-gauche laquelle ne récupère pas pour autant les voix communistes perdues.

Quant aux Verts, leur résultat régional est supérieur au total des voix Lalonde et IslerBéguin de 1994 (+ 4,9\%) soit 8,83\%) mais toujours inférieur aux voix Waechter de 1989 $(9,32 \%)$, inférieur aussi à la moyenne nationale $(-0,88 \%)$. Ce résultat est à l'unisson des élections du cycle ouvert en 1995 : les écologistes y souffrent d'un déficit persistant, comme si leur électorat urbain (sauf dans les métropoles comme Montpellier) d'autrefois avait rejoint durablement les socialistes. La carte de leur électorat est ainsi plus rurale qu'autre chose avec une zone de force relative dans le triangle Florac-Montpellier-Olargues où les meilleurs scores sont souvent dans de toutes petites communes (comme St-Énimie, Lanuéjols, Joncels). Dans ces "hauts-cantons" et ce piémont languedocien, ils sont la réplique exacte du vote Chasse-pêche-Tradition dans le registre "qualité de la vie". En cela ils reflètent la tendance nationale où le vote Vert semble intéresser les petites communes d'une part et les villes de plus de $\mathbf{1 0 0 . 0 0 0}$ habitants souvent universitaires d'autre part. De ce dernier point de vue, le vote de Mont- pellier $(10,41 \%)$ est encore loin de celui de Grenoble $(17,2 \%)$ ou de Strasbourg (16,8\%). Moins encore que le PC, les Verts ne semblent pas parvenir d'une élection à l'autre à s'implanter de manière équilibrée sur le territoire régional. Et leur électorat est sans doute l'un des plus composites et flottants de celui de la gauche plurielle.

Reste l'extrême-gauche. Avec 4,72\%, elle atteint presque sa moyenne nationale $(-0,46 \%)$ et double le pourcentage réalisé par Arlette Laguiller en 1994. Cela peut s'expliquer par l'alliance avec Alain Krivine dont la formation a été de tout temps mieux implantée dans cette région que celle de Lutte ouvrière. Le fait que le score record soit à Montpellier (5,96\%) semblerait pouvoir le confirmer. Partout où la comparaison est possible, les résultats de ces européennes sont supérieurs à ceux des régionales et des cantonales de 1998 où les deux formations s'étaient présentées séparément. Cependant et en dehors du cas de Montpellier et peut être dans certaines communes des PyrénéesOrientales, ces résultats ne semblent pas mordre réellement sur le PC. Comme au niveau national, il semble possible d'imaginer que l'extrême-gauche récupère marginalement une partie de l'électorat Front national.

De ce panorama, il ressort que la gauche dans son ensemble s'ordonne autour d'un pourcentage stable à condition qu'elle puisse à travers ses différentes composantes attirer et conserver à la fois des électeurs traditionnels revenus du vote protestataire et des nouveaux "catalo-languedociens" qui viennent s'installer de plus en plus nombreux dans la région. Du coup, les chances électorales que la gauche doit confirmer dépendent et de la formule politique nationale de la "gauche plurielle" scellée par la coalition gouvernementale et des gestions locales à la fois 


\section{Les élections européennes du 13 juin 99 en L.-R.}

départementales et municipales. Les prochaines élections municipales seront donc essentielles pour analyser les traductions de cette combinatoire dans l'espace régional.

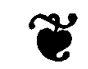

Les prochaines élections municipales seront précieuses pour observer la résolution d'un rapport de forces en voix instable jusqu'ici, même si légèrement à l'avantage de la gauche quand celle-ci se présentera groupée et plurielle aux électeurs. Ce sera la consultation où pourra se mesurer la stratégie imposée par J. Blanc et ses relais départementaux aux directions nationales produit un "retour sur investissement". L'électorat suivra-t-il la confirmation locale des alliances du sommet régional ? L'affaiblisse- ment de la triangulation devenue coutumière tant que l'extrême-droite était forte et unie permettra-t-elle non seulement de sauver les villes à première vue menacées (Carcassonne, Narbonne, Béziers, Alès, etc.) mais d'imposer durablement aux électeurs une stratégie d'extrémisation de la droite?

Quant à la gauche, qui aura aussi à affronter les élections cantonales, c'est dans sa capacité à renouveler ses programmes municipaux comme ses candidatures ainsi qu'à crédibiliser localement l'alliance gouvernementale qu' elle pourra faire de ce scrutin une nouvelle étape dans sa recomposition régionale.

C'est déjà dire combien cette prise en compte du territoire dans les élections quelles qu'elles soient est une donnée nécessaire à la compréhension rigoureuse des tendances qui travaillent l'électorat.

\begin{tabular}{|c|c|c|c|c|c|}
\hline \multirow[b]{3}{*}{ Hollande - PS } & \multirow{3}{*}{$\begin{array}{c}\text { Roussil } \\
\text { Aude } \\
31,10\end{array}$} & \multirow{3}{*}{$\begin{array}{c}\text { Resulte } \\
\text { Gard } \\
19,13\end{array}$} & \multirow{3}{*}{$\begin{array}{c}\text { Hérault } \\
21,14\end{array}$} & pales I & \\
\hline & & & & \multicolumn{2}{|c|}{ Lozère Pyrénées-O. } \\
\hline & & & & 18,13 & 21,37 \\
\hline Hue - PCF & 8,52 & 10,01 & 7,92 & 5,39 & 8,69 \\
\hline Cohn-Bendit - Verts & 7,20 & 8,46 & 10,41 & 7,97 & 7,54 \\
\hline Laguiller - LO/LCR & 4,55 & 4,81 & 4,83 & 4,03 & 4,63 \\
\hline Pasqua - RPFIE & 12,22 & 14,90 & 13,84 & 16,18 & 14,04 \\
\hline Sarkozy - RPR/DL & 8,85 & 9,31 & 8,95 & 20,21 & 9,90 \\
\hline Bayrou - UDF & 5,10 & 6,74 & 6,31 & 9,86 & 8,26 \\
\hline Le Pen - FN & 5,01 & 7,43 & 6,08 & 3,16 & 7,17 \\
\hline Mégret - MN & 3,12 & 5,69 & 4,74 & 2,24 & 4,43 \\
\hline St Josse - CPNT* & 10,09 & 8,86 & 10,90 & 8,52 & 9,03 \\
\hline Divers & - & - & - & - & - \\
\hline \multicolumn{6}{|c|}{$\begin{array}{l}\text { "Nous avons ici ajouté les pourcentages des deux listes qui revendiquaient l'electorat CPNT. Une conduite par le } \\
\text { conseiller sortant Georges Cabannes et l'autre mené par Jean Benoît. }\end{array}$} \\
\hline
\end{tabular}

